\title{
Chemical and photo-chemical bonding of polymer and silica fibers
}

\author{
Miłosz S. Chychłowski ${ }^{1}$, Sławomir Ertman ${ }^{1}$ and Tomasz R. Woliński ${ }^{1}$ \\ ${ }^{l}$ Faculty of Physics, Warsaw University of Technology, Koszykowa 75, 00-662 Warszawa,
}

Received September 24, 2014; accepted September 30, 2014; published September 30, 2014

\begin{abstract}
In this paper we present a permanent way of connecting two different fiber types. One of them is a novel fiber based on polymer as the main construction material, with a build-in photonic structure in its cladding. The second type is a typical silica single-mode fiber. Due to the differences of base materials, thermal bonding is impossible in order to keep both structures intact. However, chemical bonding by using appropriate materials can connect two types of fibers without disturbing their inner structures. The additional advantage of the proposed chemical "splicing" method is that an adhesive can act as an index matching material decreasing losses.
\end{abstract}

For the last decade, there has been a growing interest in polymer fibers due to their numerous advantages and application possibilities. Polymer photonic crystal fibers (PCFs) are similar in their structure to silica-based photonic crystal fibers characterized by a periodic lattice with a defect acting as a core [1-4].

There are several methods of connecting of two fibers. To the most common ones belong mechanical methods, in which both fibers are terminated with connectors (e.g. FC, SMA or ST) and then connected by an adapter, and also thermal methods, in which both fibers are being spliced. The splicing method requires similar melting temperatures of the spliced fibers. This condition is fulfilled for most silica and doped-silica fibers, but in the case of fused silica and polymer materials the melting temperatures are totally different: $1500^{\circ} \mathrm{C}$ and $180^{\circ} \mathrm{C}$, respectively.

Polymer photonic crystal fibers used in our experiments were produced by KIRIAMA (Fig.1): single-mode SM340 and multi-mode FM-340. The SM-340 polymer photonic crystal fiber is characterized by an $8-\mu \mathrm{m}$ core diameter, $5-\mu \mathrm{m}$ holes and $8-\mu \mathrm{m}$ holes spacing, polymethylmethacrylate (PMMA) core and cladding material with polycarbonate coating (PC). The FM-340 polymer photonic crystal fiber is characterized by a 16 $\mu \mathrm{m}$ core diameter, $5-\mu \mathrm{m}$ holes and $8-\mu \mathrm{m}$ holes spacing, polymethylmethacrylate (PMMA) core and a cladding material with polycarbonate coating (PC).

Also another custom-designed Kiriama fiber was made of cyclo-olefin polymer (Zeonex 480R). The outer- and the core- diameters of the manufactured fiber are equal to $225 \mu \mathrm{m}$ and $55 \mu \mathrm{m}$, respectively. The diameter of the single hole varies in a range of $5.3-5.8 \mu \mathrm{m}$, while the lattice constant, holes spacing $\Lambda=8.0-8.4 \mu \mathrm{m}$ (Fig. 2).
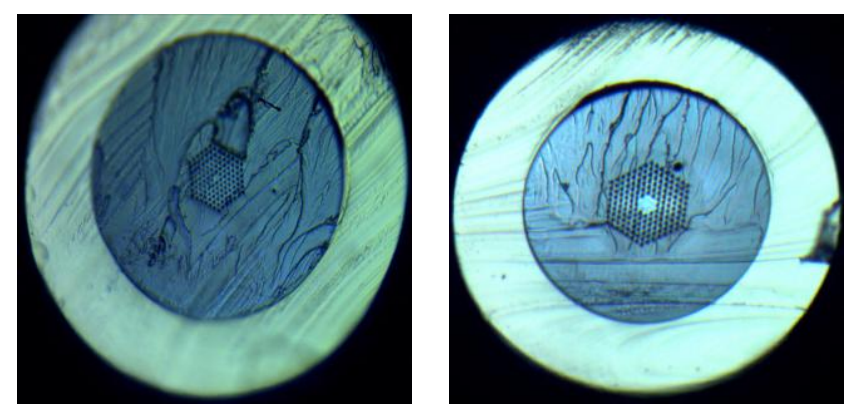

Fig. 1. KIRIAMA polymer photonic crystal fibers: single-mode SM-340 (left) and multi-mode FM-340 (right).

Alternatively, a polymer PCF i.e. polycarbonate (PC)based fiber made by Maria Curie Skłodowska University (MCSU), Lublin, Poland was characterized by a $10-\mu \mathrm{m}$ core diameter, $8-\mu \mathrm{m}$ holes, and $10-\mu \mathrm{m}$ holes spacing.

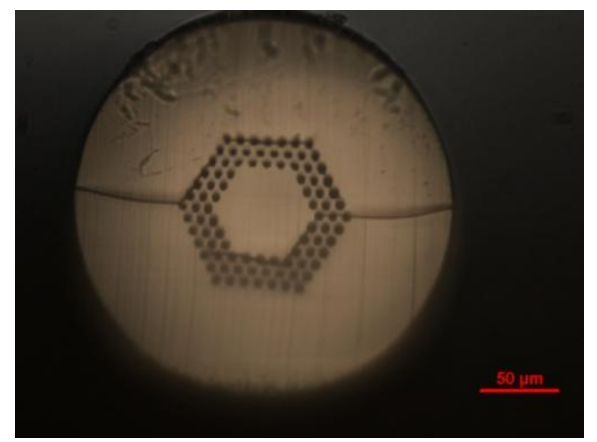

Fig. 2. Zeonex 480R polymer photonic crystal fiber (KIRIAMA).

All types of polymer PCFs were connected to the silica SM800 single-mode fiber manufactured by FIBERCORE.

The adhesives used for the "cold" splicing method were produced by Drei Bond, DYMAX along with commonly available adhesives POXIPOL and SUPER GLUE. Several types of Drei Bond adhesives were tested, such as 6020 and 6023 characterized by the viscosity 2500 [mPa.s] and $120[\mathrm{mPa} \cdot \mathrm{s}]$, respectively. DYMAX OP-420632-GEL adhesive (gel-type UV curing) is characterized by the viscosity of $57000[\mathrm{mPa} \cdot \mathrm{s}]$ and the refractive index of 1.55 . We used the DYMAX BLUEWAVE 75 UV light source for adhesives curing. As a light source for power loss measurement we used a laser with a 780nm peak wavelength and a NewPort model 1918-c served as a power meter. 
The first step of the "cold" splicing procedure is a clean cut of the polymer photonic crystal fiber and a typical cut of the single-mode silica fiber. A preliminary alignment of these two fiber types is made by using nano-precision ThorLabs stages with the help of a digital microscope. The whole setup is described in details by Ertman et al. [5]. After that an adhesive is manually placed on the fibers, and correction adjustment is made. At this point, the change is measured in transmitted power, referred to as "Power after applying adhesive" in the results presented here. Light propagation is examined by a digital microscope at the outgoing face of the fiber during preliminary adjustment procedures, then the only outgoing power is measured by a power meter. The duration of UV irradiation of $20 \mathrm{~s}$ - if required - is repeated until there are no significant power changes in fiber movement (Fig. 3). The change in transmitted power between applying and hardening of an adhesive is referred as attenuation. The distinction between power change after applying adhesive and attenuation is needed due to the fact that an adhesive acts as an index matching material at the first step of "cold" splicing.

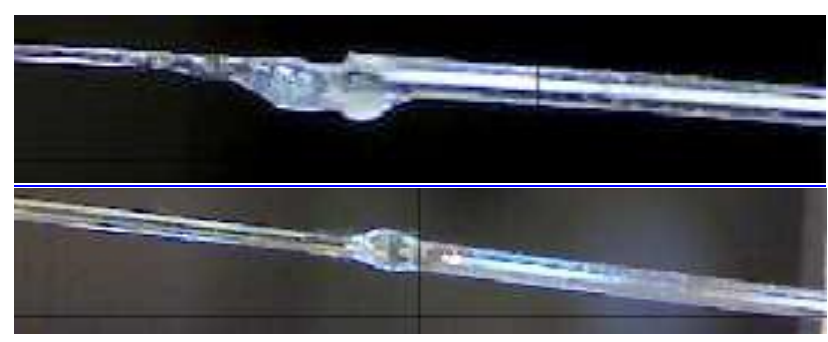

Fig. 3. "Cold" splice polymer and silica fiber top (image at top) and side (image at bottom) view.

The first attempts with "cold" splicing were made with the SM-340 photonic polymer fiber (about 1-m-long) cleaved with a razor blade. By using the 6020 adhesive, during UV curing power drastically decreased and the measured "splice" attenuation was 9.2dB. Next, the polymer FM-340 fiber was used with the same adhesive. Attenuation lowered to $5.8 \mathrm{~dB}$ but still was significant. The adhesive was replaced by two typical adhesives "Super Glue" (4.8dB) and then "POXIPOL" (0.3dB). This experiment confirmed stress-induced dis-alignment of the fibers, which, due to a rapid hardening process, cannot be compensated - Drei Bond 6020 and "Super Glue" had a hardening time from few seconds up to 20 seconds, whereas with "POXIPOL" it is a few minutes during which the dis-alignemt can be compensated. The "POXIPOL" glue was not durable enough and too much time-consuming to meet the requirements of "cold" splicing fibers. Another UV-curable adhesive with a high density was chosen to reduce stresses induced during UV irradiation.
While the PC-based polymer PCF was connected to the SM800 fiber by using a GEL adhesive for the "cold" splice, we observed the lowest average attenuation $0.8 \mathrm{~dB}$.

A GEL-type adhesive does not change the parameters of a "cold" spliced fiber though, as most liquids can infiltrate photonic structure (Fig. 4).
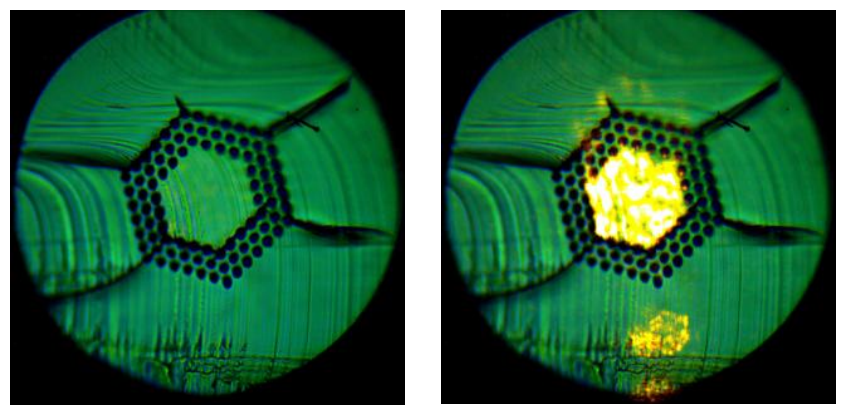

Fig. 4: Face of ZEONEX fiber (left), propagation induced in "cold" spliced system (right).

An increase in transmitted intensity was observed after applying the adhesives procedure. We can attribute the observed increase of transmitted power to refractive index matching between the two fibers. The refractive index of DYMAX OP-4-20632-GEL adhesive is equal to 1.502, which is close to the refractive index of polymer PMMA fiber 1.491 [6] and lower than PC 1.584 [7], ZEONEX 480R 1.525 but higher than that of the silica glass 1.4536 [8].

A fine-tuned procedure was based on lowering the intensity of the UV lamp and making several series of UV irradiation with time reduced to $10 \mathrm{~s}$ and fine-adjustment of fibers alignment. This improved "cold" splicing, due to the possibility of corrective adjustment, finally decreased the attenuation.

The results obtained are summarized in Tabs. 1-5.

Tab.1. Results of "cold" splicing of the SM-340 polymer PCF with the silica fiber average values for attenuation and power increase/decrease due to applied adhesive (* - best result).

\begin{tabular}{|l|c|c|}
\hline & $\begin{array}{c}\text { Power after applying } \\
\text { adhesive[\%] }\end{array}$ & Attenuation [dB] \\
\hline Drei Bond 6020 & $70 \%$ & 9.2 \\
\hline $\begin{array}{l}\text { DYMAX OP-4- } \\
\text { 20632-GEL }\end{array}$ & $570 \%$ & $3\left(0.1^{*}\right)$ \\
\hline
\end{tabular}

Tab.2. Results of "cold" splicing of the FM-340 polymer PCF with the silica fiber average values for attenuation and power increase/decrease due to applied adhesive.

\begin{tabular}{|l|c|c|}
\hline & $\begin{array}{c}\text { Power after applying } \\
\text { adhesive [\%] }\end{array}$ & Attenuation [dB] \\
\hline Drei Bond 6020 & $110 \%$ & 5.8 \\
\hline POXIPOL & $90 \%$ & 0.3 \\
\hline SUPER GLUE & $120 \%$ & 4.8 \\
\hline
\end{tabular}


Tab.3. Results of "cold" splicing of the ZEONEX polymer PCF with the silica fiber average values for attenuation and power increase/decrease due to applied adhesive.

\begin{tabular}{|l|c|c|}
\hline & $\begin{array}{c}\text { Power after applying } \\
\text { adhesive [\%] }\end{array}$ & Attenuation [dB] \\
\hline $\begin{array}{l}\text { DYMAX OP-4-20632- } \\
\text { GEL }\end{array}$ & $150 \%$ & 0.3 \\
\hline
\end{tabular}

Tab. 4. Results of "cold" splicing of the PC-based PCF with the silica fiber average values for attenuation and power increase/decrease due to applied adhesive.

\begin{tabular}{|l|c|c|}
\hline & $\begin{array}{c}\text { Power after applying } \\
\text { adhesive [\%] }\end{array}$ & Attenuation [dB] \\
\hline $\begin{array}{l}\text { DYMAX OP-4-20632- } \\
\text { GEL }\end{array}$ & $180 \%$ & -0.2 \\
\hline
\end{tabular}

Tab. 5. Results of "cold" splicing of the ZEONEX polymer PCF with the silica photonic fiber average values for attenuation and power decrease due to applied adhesive.

\begin{tabular}{|l|c|c|}
\hline & $\begin{array}{c}\text { Power after applying } \\
\text { adhesive [\%] }\end{array}$ & Attenuation [dB] \\
\hline $\begin{array}{l}\text { DYMAX OP-4-20632- } \\
\text { GEL }\end{array}$ & $70 \%$ & 3.1 \\
\hline DYMAX OP-4-20632 & $60 \%$ & 0.6 \\
\hline Drei Bond 6020 & $80 \%$ & 12.3 \\
\hline
\end{tabular}

The best results were achieved by using the DYMAX OP-4-20632-GEL adhesive (Tab. 1). It is the slowest UV curing among tested which is its greatest advantage. Other UV-curing adhesives introduced a dis-alignment of the fibers during the procedure without any ability to re-adjust the dis-aligned fibers (Tab. 2). It was caused by stresses induced while the adhesive was hardening. A similar disadvantage was presented by the SUPER GLUE - an UV curable adhesive. The slowest hardening time was obtained by using the POXIPOL glue, also non UV curable adhesive; it allowed fine adjustment but it does not have a sufficient durability in comparison to UV curable adhesives for "cold" fiber splicing. The same adhesive was used for "cold" splicing of the ZOENEX and PC fibers with a typical power increase after applying the OP-4-20632-GEL and extremely low attenuation (Tab. 3). The "cold" splice PC PCF and silica fiber have a negative value of attenuation, it can be caused by a change of the refractive index of an adhesive after UV curring inside PC PCF, and not changing the propagation mechanism - modified total internal reflection (based on both materials refractive indices). An increase in the refractive index in a photonic structure of PC PCF from 1 to 1.55 can improve its propagation properties (Tab. 4). Additionally, ZOENEX were "cold" spliced with a typical photonic crystal fiber, characterized by power loss after applying all adhesives. This can be the effect of an adhesive filling both photonic structures inside silica photonic fiber and ZEONEX PCF causing an inversion of refractive indices proportion. High attenuation for Drei Bond adhesive and relatively low attenuation for DYMAX adhesives can be caused by the photonic band gap light propagation method on an adhesive filled part of the silica fiber (Tab. 5).

In this study, we have demonstrated a "cold" splicing method of polymer PCFs and silica fibers. Several adhesives were compared and tested, including UV- and non-UV-curable adhesives. DYMAX OP-4-20632-GEL appeared to be the best solution which allowed precise adjustment during the UV curing procedure.

This work was supported by the Polish National Science Centre (NCN) under the grant No. 2011/01/B/ST7/05015.

\section{References}

[1] P.St.J. Russell, J.C. Knight, T.A. Birks, B.J. Mangan, W.J. Wadsworth, Science 299, 358 (2003).

[2] J.C. Knight, Nature 424, 847 (2003).

[3] S. Ertman, A. H. Rodríguez, M.M. Tefelska, M.S. Chychłowski, D. Pysz, R. Buczyński, E. Nowinowski-Kruszelnicki, R Dąbrowski, T.R. Woliński, J. Lightwave Techn. 30, 1208 (2012).

[4] P. Mergo, M. Gil, K. Skorupski, J. Klimek, G. Wójcik, J. Pędzisz, J. Kopec, K. Poruraj, L. Czyzewska, A. Walewski, A. Gorgol, Phot. Lett. Poland 5, 170 (2013)

[5] S. Ertman et al., submitted to Phot. Lett. Poland, 6(3), (2014).

[6] J.M. Cariou, J. Dugas, L. Martin, P. Michel, Appl. Opt. 25(3), 334 (1986); http://dx.doi.org/10.1364/AO.25.000334

[7] T. Kurokawa, N. Takato, Y. Katayama, Appl. Opt. 19(18), 3124 (1980); http://dx.doi.org/10.1364/AO.19.003124

[8] R. Kitamura, L. Pilon, M. Jonasz, Appl. Opt. 46(33), 8118 (2007); http://dx.doi.org/10.1364/AO.46.008118 